\title{
HOMOLOGICAL INDEPENDENCE OF INJECTIVE HULLS OF SIMPLE MODULES OVER CERTAIN NONCOMIMUTATIVE RINGS
}

\author{
JONATHAN S. GOLAN
}

\begin{abstract}
We study noncommutative rings $\boldsymbol{R}$ having the property that there is no nonzero homomorphism between injective hulls of nonisomorphic simple left $\boldsymbol{R}$-modules. For rings satisfying the condition that the torsion theory cogenerated by the injective hull of a simple left $R$-module is always jansian, we characterize this property in terms of the lattice of torsion theories on the category of left $\boldsymbol{R}$-modules.
\end{abstract}

0. Background and notation. Throughout the following $R$ will denote an associative (but not necessarily commutative) ring with unit element 1 and $R$-mod will denote the category of unitary left $R$-modules and module homomorphisms. Morphisms in $R$-mod will be written as acting on the right. If $M$ is an object of $R$-mod then the injective hull of $M$ will be denoted by $E(M)$. We will select, once and for all, a complete set of representatives of isomorphism classes of simple objects of $R$-mod and will denote this set by $R$-simp.

The complete brouwerian lattice of all (hereditary) torsion theories on $R$-mod will be denoted by $R$-tors. Notation and terminology concerning $R$-tors will follow [3]. In particular, if $M$ is an object of $R-\bmod$ then $\xi(M)$ will denote the smallest torsion theory in $R$-tors relative to which $M$ is torsion and $\chi(M)$ will denote the largest torsion theory in $R$-tors relative to which $M$ is torsionfree. (Note that this is precisely the torsion theory cogenerated by $E(M)$.) The unique minimal element of $R$-tors is then $\xi=\xi(0)$ and the unique maximal element of $R$-tors is $\chi=\chi(0)$. The lattice operations on $R$-tors are defined as follows: if $U \subseteq R$-tors then a left $R$-module is $\wedge U$-torsion if and only if it is torsion with respect to every member of $U$; the module is $\bigvee U$-torsionfree if and only if it is torsionfree with respect to every member of $U$. If $M$ is an object of $R$-mod and if $\tau \in R$-tors then the unique largest $\tau$-torsion submodule of $M$ will be denoted by $T_{\tau}(M)$. Note that $M / T_{\tau}(M)$ is then $\tau$-torsionfree.

The class of all objects of $R$-mod torsion with respect to a given element $\tau$ of $R$-tors is closed under taking submodules, homomorphic images, extensions, and arbitrary direct sums. It is not in general closed under taking injective hulls; when this condition holds, the torsion theory is said to be stable. It is also not in general

Received by the editors March 26, 1980.

1980 Mathematics Subject Classification. Primary 16A45, 16A53, 16A63; Secondary 06D20, 16A08, 16A51, $18 \mathrm{E} 40$.

Key words and phrases. $\boldsymbol{H}$-ring, torsion theory, simple module, jansian torsion theory, homological independence, Stone lattice. 
closed under taking arbitrary direct products; when this condition holds, the torsion theory is said to be jansian. Stable and jansian torsion theories are both amply studied in [3].

1. $H$-rings. Following [6], we say that a ring $R$ is a left $H$-ring if and only if $\operatorname{Hom}_{R}\left(E\left(M_{1}\right), E\left(M_{2}\right)\right)=0$ for any pair of distinct elements $M_{1}$ and $M_{2}$ of $R$-simp. Commutative $H$-rings have recently been studied by Camillo [1], who showed that rings regular in the sense of von Neumann, polynomial rings in finitely-many indeterminates over a field, and the ring of continuous functions on the unit interval are all of this type. Polynomial rings in infinitely-many indeterminates over a field are not $H$-rings. If $M_{1}$ and $M_{2}$ are distinct elements of $R$-simp (where $R$ is no longer assumed to be commutative) then $\operatorname{Hom}_{R}\left(M_{1}, E\left(M_{2}\right)\right)=0$ and so a sufficient condition for $R$ to be a left $H$-ring is that the torsion theory $\chi(M)$ be stable for every $M \in R$-simp. Examples of noncommutative rings having this property can be found, for instance, in [5]. Moreover, left $Q I$-rings (i.e., those rings over which every quasi-injective left $R$-module is injective) and left $V$-rings (those rings over which every simple left $\boldsymbol{R}$-module is injective) are clearly left $\boldsymbol{H}$-rings. These rings and their applications are discussed in [2].

\section{(1.1). Proposition. Any factor ring of a left $H$-ring is a left $H$-ring.}

Proof. Let $R$ be a left $H$-ring and let $S=R / I$ be a factor ring of $R$. Then $S$-simp $=\{M \in R$-simp $\mid I M=0\}$ is a complete set of representatives of the isomorphism classes of simple left $S$-modules [6]. Moreover, if $N_{1}$ and $N_{2}$ are two left $S$-modules then $\operatorname{Hom}_{R}\left(N_{1}, N_{2}\right)=\operatorname{Hom}_{S}\left(N_{1}, N_{2}\right)$; if $N$ is a left $S$-module then the injective hull of $N$ in $S$-mod is $E^{\prime}(N)=\left\{x \in E\left({ }_{R} N\right) \mid I x=0\right\}$ [6, Proposition 2.27].

Now let $M_{1}$ and $M_{2}$ be distinct elements of $S$-simp and, for $i=1,2$, let $E^{\prime}\left(M_{i}\right)$ be the injective hull of $M_{i}$ in $S$-mod. Then each $E^{\prime}\left(M_{i}\right)$ is an $R$-submodule of $E\left(M_{i}\right)$ and so, if there exists a nonzero $S$-homomorphism from $E^{\prime}\left(M_{1}\right)$ to $E^{\prime}\left(M_{2}\right)$ then it can be extended to a nonzero $R$-homomorphism from $E\left(M_{1}\right)$ to $E\left(M_{2}\right)$, which is impossible since $R$ is a left $H$-ring. Thus $S$ too must be a left $H$-ring.

(1.2). Proposition. If $R$ is a ring satisfying the condition that $T_{\chi\left(M_{1}\right)}(R)+$ $T_{\chi\left(M_{2}\right)}(R)=R$ for any pair $M_{1}, M_{2}$ of distinct elements of $R$-simp then $R$ is a left $H$-ring. The converse holds if $R$ satisfies the additional condition that $\chi(M)$ is jansian for every $M \in R$-simp.

Proof. Let $M_{1}$ and $M_{2}$ be distinct elements of $R$-simp and assume that $0 \neq \alpha \in \operatorname{Hom}_{R}\left(E\left(M_{1}\right), E\left(M_{2}\right)\right)$. Since $R=T_{\chi\left(M_{1}\right)}(R)+T_{\chi\left(M_{2}\right)}(R)$, we can write $1=a+b$, where $a \in T_{\chi\left(M_{1}\right)}(R)$ and $b \in T_{\chi\left(M_{2}\right)}(R)$. But $\operatorname{Hom}_{R}\left(T_{\chi\left(M_{1}\right)}(R), E\left(M_{i}\right)\right)$ $=0$ for $i=1,2$ and so, in particular, $T_{\chi\left(M_{i}\right)}(R)=\left(0: E\left(M_{i}\right)\right)$ for $i=1$, 2. Thus for any $x \in E\left(M_{1}\right)$ we have $x \alpha=(1 x) \alpha=(a x+b x) \alpha=(b x) \alpha=b(x \alpha)=0$, which contradicts the choice of $\alpha$. Therefore $R$ is a left $H$-ring.

Now, conversely, assume that $R$ is a left $H$-ring satisfying the condition that $\chi(M)$ is jansian for every $M \in R$-simp. Let $M_{1}$ and $M_{2}$ be distinct elements of $R$-simp and set $I=T_{\chi\left(M_{1}\right)}(R)+T_{\chi\left(M_{2}\right)}(R)$. Assume that this is a proper ideal of $R$. Then $I$ is contained in a maximal left ideal $H$ of $R$. Moreover, for $i=1,2$ we see 
that $R / T_{\chi\left(M_{i}\right)}(R)$ is $\chi\left(M_{i}\right)$-torsionfree and so there exists an index set $\Omega(i)$ and an $R$-monomorphism from $R / T_{\chi\left(M_{i}\right)}(R)$ into $E\left(M_{i}\right)^{\Omega(i)}$. If $\nu_{i}: R / T_{\chi\left(M_{i}\right)}(R) \rightarrow R / H$ is the canonical surjection then, by injectivity, there exists an $R$-homomorphism $\alpha_{i}$ making the diagram

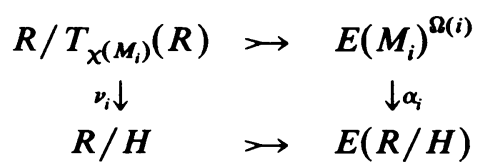

commute. In particular, this implies that $E\left(M_{i}\right)^{\Omega(i)}$ is not $\chi(R / H)$-torsion. Since $\chi(R / H)$ is jansian, this implies that $E\left(M_{i}\right)$ is not $\chi(R / H)$-torsion for $i=1,2$ and so $\operatorname{Hom}_{R}\left(E\left(M_{i}\right), E(R / H)\right) \neq 0$ for $i=1,2$. This contradicts the hypothesis that $R$ is a left $\boldsymbol{H}$-ring.

Since the lattice $R$-tors is complete brouwerian, every $\tau \in R$-tors has a meet pseudocomplement which, following the notation of [3], we will denote by $\tau^{\perp}$. Then $\tau \wedge \tau^{\perp}=\xi$ and $\tau^{\perp} \geqslant \tau^{\prime}$ for every torsion theory $\tau^{\prime} \in R$-tors satisfying the condition $\tau \wedge \tau^{\prime}=\xi$. Tèbyrcè [7] has given a complete characterization of $\tau^{\perp}$ as follows: if we denote the set of all $\tau$-torsion elements of $R$-simp by $\tau$-simp then $\tau^{\perp}=\bigwedge\{\chi(M) \mid M \in \tau$-simp $\}$.

(1.3). Proposition. A ring $R$ is a left $H$-ring if and only if $\xi(E(M))^{\perp}=\xi(M)^{\perp}$ for every $M \in R$-simp.

Proof. If $M_{1}$ and $M_{2}$ are distinct elements of $R$-simp and if $0 \neq \alpha \in$ $\operatorname{Hom}_{R}\left(E\left(M_{1}\right), E\left(M_{2}\right)\right)$ then $E\left(M_{1}\right) \alpha$ is a nonzero submodule of $E\left(M_{2}\right)$ and so, by simplicity, $M_{2} \subseteq E\left(M_{1}\right) \alpha$. This implies that $M_{2}$ is $\xi\left(E\left(M_{1}\right)\right)$-torsion. On the other hand, if $M_{2}$ is $\xi\left(E\left(M_{1}\right)\right)$-torsion then there exists a nonzero $R$-homomorphism from $E\left(M_{1}\right)$ to $E\left(M_{2}\right)$. Thus we see that the condition that $R$ be a left $H$-ring is equivalent to the condition that $\xi(E(M))$-simp $=\{M\}$ for any simple left $R$-module $M$ or, in other words, that $\xi(E(M))^{\perp}=\chi(M)$ for all such $M$. But, by Tèbyrcè's characterization, it is immediate that $\chi(M)=\xi(M)^{\perp}$ for any simple left $R$-module $M$, and so we are done.

Recall that a lattice $L$, every element $u$ of which has a meet pseudocomplement $u^{\perp}$, is said to be a Stone lattice if and only if $\left(u_{1} \wedge u_{2}\right)^{\perp}=u_{1}^{\perp} \vee u_{2}^{\perp}$ for all $u_{1}$, $u_{2} \in L$. Stone lattices are an important class of distributive lattices and have been fully characterized. See [4] for details.

(1.4). Proposition. If $R$ is a left $H$-ring satisfying the condition that $\chi(M)$ is jansian for every $M \in R$-simp then $R$-tors is a Stone lattice.

Proof. If $\tau$ and $\sigma$ are two elements of $R$-tors then $\tau \wedge \sigma<\tau$ and so $\left.(\tau \wedge \sigma)^{\perp}\right\rangle$ $\tau^{\perp}$. Similarly $(\tau \wedge \sigma)^{\perp} \geqslant \sigma^{\perp}$ and so $(\tau \wedge \sigma)^{\perp} \geqslant \tau^{\perp} \vee \sigma^{\perp}$. Assume that this inequality is strict. Then there exists a nonzero left $R$-module $N$ which is $(\tau \wedge \sigma)^{\perp}$ torsion and $\left(\tau^{\perp} \vee \sigma^{\perp}\right)$-torsionfree. That is to say,

(1) $\operatorname{Hom}_{R}(N, E(M))=0$ for all $M \in \tau$-simp $\cap \sigma$-simp;

(2) there exists an index set $\Omega$ and an $R$-monomorphism

$$
\phi: N \rightarrow\left(\prod\{E(M) \mid M \in \tau \text {-simp }\}\right)^{\Omega} ;
$$


(3) there exists an index set $\Lambda$ and an $R$-monomorphism

$$
\psi: N \rightarrow\left(\prod\{E(M) \mid M \in \sigma-\operatorname{simp}\}\right)^{\Lambda} .
$$

In particular, from (1) we see that $N \phi$ is contained in

$$
E_{1}=\left(\prod\{E(M) \mid M \in \tau-\operatorname{simp} \backslash \sigma \text {-simp }\}\right)^{\Omega}
$$

and $N \psi$ is contained in

$$
E_{2}=\left(\prod\{E(M) \mid M \in \sigma \text {-simp } \backslash \tau \text {-simp }\}\right)^{\Lambda} .
$$

Since $E_{2}$ is injective, there exists an $R$-homomorphism $\beta: E_{1} \rightarrow E_{2}$ satisfying $\phi \beta=\psi$. Since $\chi(M)$ is jansian for every $M \in \sigma$-simp $\backslash \tau$-simp by assumption, it follows that $\chi\left(E_{2}\right)=\bigwedge\{\chi(M) \mid M \in \sigma$-simp $\backslash \tau$-simp $\}$ is also jansian. In particular, this implies that there must exist simple left $R$-modules $M_{1} \in \tau$-simp $\backslash \sigma$-simp and $M_{2} \in \sigma$-simp $\backslash \tau$-simp for which we have $\operatorname{Hom}_{R}\left(E\left(M_{1}\right), E\left(M_{2}\right)\right) \neq 0$, contradicting the hypothesis that $R$ is a left $H$-ring. This implies that we must indeed have $(\tau \wedge \sigma)^{\perp}=\tau^{\perp} \vee \sigma^{\perp}$.

(1.5). Proposition. For a ring $R$ satisfying the condition that $\chi(M)$ is jansian for every simple left $R$-module $M$ the following conditions are equivalent:

(1) $R$ is a left $H$-ring.

(2) (i) $R$-tors is a Stone lattice; and

(ii) if $M_{1}$ and $M_{2}$ are distinct elements of $R$-simp then

$$
T_{\chi\left(M_{1}\right)}(R)+T_{\chi\left(M_{2}\right)}(R)=T_{\chi\left(M_{1}\right) \vee \chi\left(M_{2}\right)}(R)
$$

Proof. By Proposition 1.4 we already know that (1) implies (2)(i). Moreover, if $M_{1}$ and $M_{2}$ are distinct elements of $R$-simp then by Proposition 1.2 we have $R=T_{\chi\left(M_{1}\right)}(R)+T_{\chi\left(M_{2}\right)}(R) \subseteq T_{\chi\left(M_{1}\right) \vee \chi\left(M_{2}\right)}(R) \subseteq R$ and so we must have (2)(ii). Conversely, assume (2). If $M_{1}$ and $M_{2}$ are distinct elements of $R$-simp then by [3, Proposition 12.1] we have $\xi=\xi\left(M_{1}\right) \wedge \xi\left(M_{2}\right)$ and so, by (2)(i), we have $\chi=\xi^{\perp}$ $=\xi\left(M_{1}\right)^{\perp} \vee \xi\left(M_{2}\right)^{\perp}=\chi\left(M_{1}\right) \vee \chi\left(M_{2}\right)$. This implies that $R=T_{\chi\left(M_{1}\right) \vee \chi\left(M_{2}\right)}(R)$ and so by (2)(ii) we have $R=T_{\chi\left(M_{1}\right)}(R)+T_{\chi\left(M_{2}\right)}(R)$. By Proposition 1.2, this implies that $R$ is a left $H$-ring.

2. The jansian condition. The "converse" portion of Proposition 1.2 is not true unless some other condition is present besides the condition that $R$ is a left $H$-ring. To see this, we note that the ring $Z$ of integers is a left $H$-ring since the simple left $\mathbf{Z}$-modules are just those of the form $\mathbf{Z} /(p)$, where $p$ is a prime integer. For each such $p, E(Z /(p))=Z\left(p^{\infty}\right)$; see [6] for details. On the other hand, since $Z$ is an integral domain, we have $T_{\tau}(Z)=0$ for all $\chi \neq \tau \in Z$-tors. (This example was supplied to me by Jay Shapiro in a private communication.)

The condition imposed in $\S 1$-namely that $\chi(M)$ is jansian for every simple left $R$-module $M$-is sufficient though probably not necessary. It is not the purpose of this note to enter in detail into the ramifications of this condition, but it is worth pointing out two extreme examples.

(1) A ring $R$ is said to be left semiartinian if and only if every nonzero left $R$-module has a nonzero socle. If $R$ is a left semiartinian ring then any torsion 
theory $\chi \neq \tau \in R$-tors is of the form $\bigwedge\{\chi(M) \mid M \in \mathbf{A}\}$ for some nonempty subset $\mathbf{A}$ of $R$-simp. As a result, if $R$ is a left semiartinian ring then every torsion theory of the form $\chi(M)$ for some simple left $R$-module $M$ is jansian if and only if every element of $R$-tors is jansian. By [3, Proposition 22.22] we see that this happens if and only if the ring $R$ is right perfect. A ring $R$ is left stable if and only if every element of $R$-tors is stable. By Proposition 1.3 , it is clear that left stable rings are left $\boldsymbol{H}$-rings. Left stable left semiartinian rings are completely characterized in [3, Proposition 23.8] and such rings need not be right perfect. Hence we see that the jansian condition is not a consequence of the condition that $R$ be a left $\boldsymbol{H}$-ring.

(2) A ring $R$ is said to be left local if and only if all simple left $R$-modules are isomorphic. If $M$ is a simple left $R$-module and if $R$ is left local then for any proper left ideal $I$ of $R$ we have a canonical epimorphism from $R / I$ to $M$ and so, in particular, $\operatorname{Hom}_{R}(R / I, E(M)) \neq 0$. Thus there are no proper $\chi(M)$-dense left ideals of $R$ and so $\chi(M)=\xi$. This torsion theory is always jansian and so the jansian condition holds for any left local ring.

ACKNOWLedgment. Portions of this note were written while the author was spending a sabbatical leave at Indiana University in Bloomington. The author wishes to thank the Department of Mathematics at Indiana University for its generous hospitality and support during his visit.

\section{REFERENCES}

1. V. Camillo, Homological independence of injective hulls of simple modules over commutative rings, Comm. Algebra 6 (1978), 1459-1469.

2. J. Cozzens and C. Faith, Simple noetherian rings, Cambridge Tracts in Math., no. 69, Cambridge Univ. Press, Cambridge, 1975.

3. J. Golan, Localization of noncommutative rings, Marcel Dekker, New York, 1975.

4. G. Grätzer, Lattice theory, W. H. Freeman, San Francisco, Calif., 1971.

5. Z. Papp, On stable noetherian rings, Trans. Amer. Math. Soc. 213 (1975), 107-114.

6. D. W. Sharpe and P. Vámos, Injective modules, Cambridge Tracts in Math., no. 62, Cambridge Univ. Press, Cambridge, 1972.

7. E. I. Tèbyrcè, The boolean nature of the lattice of torsions in modules, Mat. Issled. 8 (1973), 92-105. (Russian)

Departmient of MATHematics, University of Haifa, HAIfA, IsRael 